\title{
PRODUÇÃO E VALOR NUTRITIVO DA GRAMA BERMUDA FLORAKIRK [Cynodon dactylon (L.) Pers.] EM DIFERENTES IDADES DE CRESCIMENTO
}

\author{
Andréa Campmany Vieira ${ }^{1,3 *}$; Cláudio Maluf Haddad ${ }^{2}$; Flávio Geraldo Ferreira Castro ${ }^{1,3}$; Osvaldo \\ Rubens Peralta Heisecke ${ }^{1,4}$; João Maurício Bueno Vendramini' ${ }^{1,4}$; Vânia Maria Quecini ${ }^{1,3}$ \\ ${ }^{1}$ Pós-Graduandos do Depto. de Produção Animal - ESALQ/USP. \\ ${ }^{2}$ Depto. de Produção Animal - ESALQ/USP, C.P. 9 - CEP: 13418-900 - Piracicaba, SP. \\ ${ }^{3}$ Bolsista do CNPq. \\ ${ }^{4}$ Bolsista da CAPES. \\ *e-mail: insecta@ufla.br
}

RESUMO: O experimento foi conduzido em Itapetininga/SP, numa pastagem de Florakirk (Cynodon dactylon) formada há um ano, com o objetivo de avaliar a produção, composição químico-bromatológica e digestibilidade do cultivar em função da sua idade de crescimento. Utilizou-se o delineamento de blocos ao acaso, com 6 tratamentos (idades de crescimento definidas por cortes realizados aos 20, 30, 40, 50, 60 e 70 dias após o corte de uniformização) e 4 repetições. A produção de matéria seca da parte aérea aumentou de forma quadrática $(P<0,05)$, com valor máximo de $215 \mathrm{~g} \mathrm{~m}^{-2}$, aos 70 dias. A digestibilidade verdadeira in vitro da matéria orgânica decresceu entre 20 e 70 dias (de 711 para $549 \mathrm{~g} \mathrm{~kg}^{-1}$ ), enquanto os teores de fibra em detergente neutro e fibra em detergente ácido aumentaram de forma quadrática $(\mathrm{P}<0,05)$. Os teores de $\mathrm{N}, \mathrm{P}, \mathrm{K}, \mathrm{Mg}$ e Ca decresceram com o aumento da idade, não havendo diferença na concentração de $S$ entre 20 e 60 dias. Foi observada uma maior variação na concentração de macronutrientes que na de micronutrientes ( $\mathrm{Cu}, \mathrm{Fe}, \mathrm{Mn}$ e $\mathrm{Zn}$ ) com o aumento na idade da planta, não havendo influência da idade sobre a concentração de B na planta. No corte aos 40 dias de crescimento, observou-se a melhor combinação entre valor nutritivo e produção de matéria seca da forragem. Observou-se a ocorrência de cigarrinhas e doenças foliares, que pode ter afetado o desenvolvimento da pastagem.

Palavras-chave: idade de crescimento, valor nutritivo, composição química, produção vegetal

\section{YIELD AND NUTRITIVE VALUE OF FLORAKIRK BERMUDAGRASS [Cynodon dactylon (L.) PERS.] AT DIFFERENT PLANT AGES}

\begin{abstract}
This research was conducted in Itapetininga, SP, Brazil, using a one year-old pasture, with the objective of evaluating the effect of plant age on yield, chemical and mineral composition, and digestibility of Florakirk bermudagrass (Cynodon dactylon). The experimental design consisted of randomized complete blocks, with six treatments replicated four times. Treatments consisted of six cutting ages (20-70 days) at ten-day intervals. Dry matter yield increased quadratically $(P<0.05)$, reaching a maximum at 70 days $\left(215 \mathrm{~g} \mathrm{~m}^{-2}\right)$. In vitro organic matter digestibility decreased $\left(711 \mathrm{~g}^{\text {to }} 549 \mathrm{~g} \mathrm{~kg}^{-1}\right)$ between 20 to 70 days. Neutral detergent fiber and acid detergent fiber increased quadratically $(P<0.05)$ as age increased. $\mathrm{N}, \mathrm{P}, \mathrm{K}, \mathrm{Mg}$, and $\mathrm{Ca}$ concentration decreased with increasing age. There was no difference $(P>0.05)$ in $S$ concentration between 20 and 60 days. A larger variation for macronutrient concentrations was observed than for micronutrient ( $\mathrm{Cu}, \mathrm{Fe}, \mathrm{Mn}$, and $\mathrm{Zn}$ ) concentrations with increasing plant age. There was no influence of age on $B$ concentration. The best association of nutritive value and yield was observed at $\mathbf{4 0}$ days. Occurrence of the spittlebug and foliar diseases was observed, which probably retarded the vegetative and maturity processes.
\end{abstract}

Key words: plant age, nutritive value, chemical composition, grass yield

\section{INTRODUÇÃO}

Devido à importância do alimento volumoso na nutrição de ruminantes, tanto em termos qualitativos como quantitativos, uma vez que a maioria do rebanho bovino brasileiro é mantida em regime de pasto, observa-se uma busca constante de novas variedades forrageiras, destinadas a sistemas de pastejo ou para produção de forragem conservada (silagem ou 
feno). Dentre as espécies forrageiras, tem-se difundido o uso daquelas pertencentes ao gênero Cynodon, principalmente as gramas bermuda e as gramas estrela. A Coastcross-1 (C. dactylon) é um dos cultivares de grama bermuda mais antigos introduzidos no Brasil, seguida pela Tifton 68 (C. nlemfuensis), Tifton 85 (Cynodon spp.) e mais recentemente pelas gramas estrela Florico e Florona (C. nlemfuensis) e a grama bermuda Florakirk. Entretanto, esta introdução de cultivares no Brasil não foi precedida por trabalhos que estudassem o comportamento dos mesmos em nosso meio e que determinassem parâmetros de manejo.

A Florakirk é um híbrido desenvolvido em Tifton (Estado da Geórgia, EUA) pelo Dr. G. W. Burton; lançado comercialmente pela Universidade da Flórida (EUA) em 1994, foi destinado principalmente à produção de feno no norte da Flórida e sul da Geórgia devido às suas características de caule fino, persistência, boa produção, boa qualidade e relativa resistência ao frio (Mislevy et al., 1995).

Mislevy et al. (1995) recomendam, para a Florkirk, intervalos entre pastejos de 4 semanas e de 4 a 5 semanas entre cortes para fenação, buscando integrar qualidade e produção de forragem, sem afetar a persistência da pastagem. Em Coastcross-1, cultivar muito semelhante à Florakirk, Palhano \& Haddad (1992) observaram intervalo de corte ideal entre 30 e 40 dias.

O valor nutritivo de uma forragem é caracterizado pela sua composição química, digestibilidade e natureza dos produtos digestíveis enquanto a qualidade da forragem envolve uma avaliação integrada de seu valor nutritivo e do nível de consumo de matéria seca pelo animal (Crowder \& Chheda, 1982); assim, a produtividade animal, expressa em termos de produção de carne, leite e lã, é influenciada em parte pelo valor nutritivo do alimento consumido (Vieira \& Gomide, 1970).

Segundo Raymond (1969), conforme a planta forrageira cresce, sua produção aumenta, mas seu valor nutritivo tende a decrescer; portanto, a determinação do estádio ótimo de utilização da mesma requer a associação de dados sobre produção em diferentes estádios de maturidade e de parâmetros de valor nutritivo.

O valor nutritivo das plantas é afetado por fatores fisiológicos, morfológicos, ambientais e por diferenças entre espécies, sendo que, no caso das forragens, o declínio no valor nutritivo associado ao aumento da idade normalmente é explicado como resultado do desenvolvimento da maturidade da planta. A digestibilidade é uma importante medida do valor nutritivo de forragens, sendo o parâmetro mais utilizado na avaliação de alimentos, pois apresenta menor variação interanimal, permitindo o estabelecimento de valores alimentares relativos. A digestibilidade em gramíneas tropicais declina continuamente durante o crescimento das mesmas, sendo também afetada pela fração da planta analisada, clima, teor de proteína bruta e de minerais, entre outros fatores (Crowder \& Chheda, 1982; Van Soest, 1994).

Os níveis dos minerais na planta forrageira dependem basicamente da espécie vegetal, das condições edafo-climáticas e do estádio de maturidade da planta. Dependendo do nutriente, o teor pode aumentar, diminuir ou permanecer constante à medida que a planta atinge a maturidade (Underwood, 1971).

O objetivo deste trabalho foi avaliar o efeito da idade da planta sobre a produção, composição químico-bromatológica e digestibilidade da grama bermuda Florakirk, obtendo-se assim informações que contribuam para a utilização eficiente desta forrageira.

\section{MATERIAL E MÉTODOS}

O experimento foi conduzido no município de Itapetininga, Estado de São Paulo $\left(23^{\circ} 42^{\prime} \mathrm{S}, 48^{\circ} 04^{\prime} \mathrm{W}\right.$ e altitude de $\left.680 \mathrm{~m}\right)$ ocupando $400 \mathrm{~m}^{2}$ de uma pastagem exclusiva de Florakirk implantada um ano antes do início do ensaio e destinada à produção de feno. O clima da região é Cfa (mesotérmico úmido com verão quente e sem estação seca) e o solo do local é Podzólico Vermelho Amarelo com a seguinte composição química na camada de 0 a 20 cm: $\mathrm{pH}\left(\mathrm{CaCl}_{2}\right)=4,5 ;$ M.O. $=15 \mathrm{~g} \mathrm{dm}^{-3}$; $\mathrm{P}($ resina $)=5 \mathrm{mg} \mathrm{dm}^{-3} ; \mathrm{S}-\mathrm{SO}_{4}=8 \mathrm{mg} \mathrm{dm}^{-3}$; $\mathrm{K}=0,3 \mathrm{mmol}_{\mathrm{cm}}^{-3} ; \mathrm{Ca}=14 \mathrm{mmol}_{\mathrm{c}} \mathrm{dm}^{-3} ; \mathrm{Mg}^{-}$ $5 \mathrm{mmol}_{\mathrm{c}} \mathrm{dm}^{-3} ; \mathrm{H}+\mathrm{Al}=22 \mathrm{mmol}_{\mathrm{c}} \mathrm{dm}^{-3} ; \mathrm{CTC}=$ $41,3 \mathrm{mmol}_{\mathrm{c}} \mathrm{dm}^{-3} ; \mathrm{V} \%=46,7 ; \mathrm{B}$ (água quente) $=$ $0,32 \mathrm{mg} \mathrm{dm}{ }^{-3}$; $\mathrm{Cu}$ (DTPA) $=0,5 \mathrm{mg} \mathrm{dm}^{-3}$; $\mathrm{Fe}(\mathrm{DTPA})=18,0 \mathrm{mg} \mathrm{dm}^{-3} ; \mathrm{Mn}($ DTPA $)=1,8 \mathrm{mg}$ $\mathrm{dm}^{-3} ; \mathrm{Zn}$ (DTPA) $=3,6 \mathrm{mg} \mathrm{dm}^{-3}$.

Dezessete dias antes do corte de uniformização foram aplicados por hectare: $5 \mathrm{Mg}$ de calcário dolomítico tipo "filler", $80 \mathrm{~kg}$ de $\mathrm{N}, 320 \mathrm{~kg}$ de $\mathrm{P}_{2} \mathrm{O}_{5}, 240 \mathrm{~kg}$ de $\mathrm{K}_{2} 0,400 \mathrm{~kg}$ de $\mathrm{Ca}$, $180 \mathrm{~kg}$ de $\mathrm{Mg}, 96 \mathrm{~kg}$ de S, $2 \mathrm{~kg}$ de $\mathrm{B}, 1 \mathrm{~kg}$ de $\mathrm{Cu}, 2,4 \mathrm{~kg}$ de $\mathrm{Mn}, 0,12 \mathrm{~kg}$ de $\mathrm{Mo}$ e $11 \mathrm{~kg}$ de $\mathrm{Zn}$, tendo sido utilizados os fertilizantes sulfato de 
amônio, termofosfato magnesiano (Yoorin Master) e cloreto de potássio.

O experimento foi iniciado em 20/01/97, quando a pastagem foi submetida a um corte de uniformização, a $10 \mathrm{~cm}$ da superfície do solo, e foi conduzido durante 70 dias, quando foram realizadas amostragens na pastagem a cada 10 dias, sendo a primeira 20 dias após o corte de rebaixamento. $O$ delineamento experimental adotado foi blocos ao acaso, com 6 tratamentos (cortes aos 20, 30, 40, 50, 60 e 70 dias de crescimento da planta) e 4 repetições. As amostragens foram realizadas com o auxílio de um quadrado de ferro de $1 \mathrm{~m}^{2}(1 \mathrm{~m} \times 1 \mathrm{~m})$, arremessado aleatoriamente na área delimitada pelo bloco; a vegetação contida dentro do quadrado foi cortada com uma tesoura a $10 \mathrm{~cm}$ da superfície do solo. As áreas amostradas foram delimitadas com estacas, evitando assim novas amostragens.

Após o corte, o material amostrado foi acondicionado em saco plástico vedado e imediatamente levado ao laboratório, onde foi pesado e submetido à lavagem com água destilada e deionizada, sendo finalmente acondicionado em sacos de papel e colocado em estufa de circulação forçada a $65^{\circ} \mathrm{C}$ durante 72 horas. Após a secagem, o material foi novamente pesado e procedeu-se a moagem do mesmo em moinho tipo Wiley com peneira de $1 \mathrm{~mm}$ (AOAC, 1995).

O teor de enxofre foi determinado segundo Bataglia (1976) e para os demais nutrientes foram utilizadas as metodologias descritas por Malavolta et al. (1989). A digestibilidade verdadeira in vitro da matéria orgânica foi determinada pelo método de Tilley \& Terry (1963), modificado por Tinnimit (1974) e as análises de FDN e FDA segundo método de Goering \& Van Soest (1970). O teor de proteína bruta foi estimado através da multiplicação do teor de N por 6,25 (AOAC, 1995).

Durante o período experimental, a precipitação pluvial foi: 0 a 20 dias de crescimento $=337 \mathrm{~mm}$; de 21 a 30 dias $=$ $158 \mathrm{~mm}$; de 31 a 40 dias $=12 \mathrm{~mm}$; de 41 a 50 dias $=57 \mathrm{~mm}$; de 51 a 60 dias $=5 \mathrm{~mm}$ e de $61 \mathrm{a}$ 70 dias $=0 \mathrm{~mm}$.

Nas análises estatísticas foram utilizados os procedimentos do SAS LAB e GLM do Sistema de Análises Estatísticas SAS (SAS Institute, 1988), onde foram obtidas as análises de variância, testes de comparações entre médias e equações de regressão simples relacionando a idade de crescimento e a variável em questão.

\section{RESULTADOS E DISCUSSÃO}

Sintomas de mancha foliar e mancha foliar preta, doenças foliares causadas pelos patógenos Bipolaris spp. e Phyllachora spp., respectivamente, foram observados entre as idades de 30 e 40 dias. Tais doenças foram controladas com a aplicação de fungicida à base de tiazol (Tilt; 5 litros ha-1 $)$. A Florakirk mostrou-se suscetível ao ataque de cigarrinha das pastagens (Deois spp.) e para seu controle foi aplicado inseticida biológico à base de fungo entomopatogênico (Metarhizium anisopliae). Segundo Mislevy et al. (1995), este cultivar é suscetível à ocorrência das doenças foliares observadas, porém é resistente ao ataque de cigarrinhas. Entre 40 e 50 dias de crescimento ocorreu infestação de lagarta curuquerê-dos-capinzais (Mocis latipes), controlada através de inseticida organofosforado (Clorpirifós Etil; 1 litro ha-1).

A produção de matéria seca de Florakirk, a digestibilidade verdadeira in vitro da matéria orgânica (DVIVMO), a fibra insolúvel em detergente neutro (FDN) e a fibra insolúvel em detergente ácido (FDA) variaram de forma quadrática com o aumento na idade de crescimento $(P<0,05)$ (Figura 1).

A produção de matéria seca cresceu até os 70 dias $\left(215 \mathrm{~g} \mathrm{~m}^{-2}\right)$, segundo a equação de regressão e considerando-se apenas o intervalo de 20 a 70 dias sujeito à análise. A redução nas

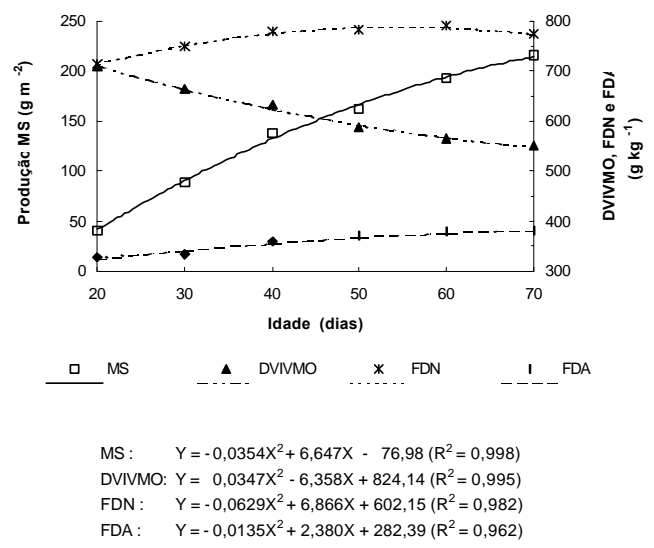

Figura 1 - Produção de matéria seca (MS), digestibilidade verdadeira in vitro da matéria orgânica (DVIVMO), fibra em detergente neutro (FDN) e fibra em detergente ácido (FDA) da parte aérea de Florakirk, em função da idade de crescimento. 
taxas de crescimento das plantas ocorre devido ao sombreamento progressivo das folhas inferiores (Gomide, 1973), tornando-as menos efetivas fotossinteticamente, e ao aumento da taxa de senescência de folhas em relação à síntese das folhas mais novas (Vickery, 1981).

Palhano \& Haddad (1992) também observaram acréscimo quadrático na produção de matéria seca com o avanço da idade, sendo o valor máximo obtido aos 60 dias $\left(916 \mathrm{~g} \mathrm{~m}^{-2}\right)$, porém os acréscimos na produção a partir de 40 dias foram pequenos, ocorrendo praticamente uma estabilização na produção, o que não foi observado na Florakirk, uma vez que até os 70 dias a produção de matéria seca não atingiu seu potencial máximo. Gomide (1996) obteve produção de matéria seca de Florakirk superior à observada

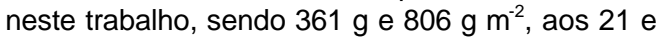
70 dias, respectivamente.

Mislevy et al. (1995) apresentaram como produção potencial de matéria seca de Florakirk, submetida a corte entre as idades de 28 a 35 dias, 3,4 a 4,5 Mg ha-1 (adubação de 78-34-67 kg ha ${ }^{-1}$ $\mathrm{ano}^{-1}$ de $\mathrm{N}-\mathrm{P}_{2} \mathrm{O}_{5}-\mathrm{K}_{2} \mathrm{O}$ e micronu-trientes). Apesar das adubações realizadas e das condições climáticas favoráveis, a produção observada neste trabalho atingiu apenas $25 \%$ deste potencial. A baixa produção de matéria seca pode ser resultado da ocorrência de cigarrinha das pastagens no início do crescimento ou, ainda, devido à ocorrência de doenças.

Os valores máximos e mínimos de DVIVMO foram observados aos 20 dias $(711 \mathrm{~g}$ $\mathrm{kg}^{-1}$ ) e aos 70 dias (549 $\mathrm{g} \mathrm{kg}^{-1}$ ), respectivamente. $\mathrm{O}$ decréscimo na digestibilidade com o aumento da idade da planta também foi observado em diversos cultivares de grama bermuda submetidos a corte (Monson \& Burton, 1982; Holt \& Conrad, 1986) ou a pastejo (Mislevy et al., 1988; Adjei et al., 1989; Pedreira, 1995). Este decréscimo deve-se ao aumento no conteúdo de parede celular (o que pode ser observado pela crescente concentração de FDN, conforme Figura 1), associado, provavelmente, a um aumento na lignificação da mesma.

A DVIVMO decresceu numa taxa de 3,2 $\mathrm{g} \mathrm{kg}^{-1} \mathrm{dia}^{-1}$, inferior à citada por Crowder \& Chheda (1982) para gramíneas tropicais (5 a $6 \mathrm{~g} \mathrm{~kg}^{-1} \mathrm{dia}^{-1}$ ) e à observada em Coastcross-1 (Palhano \& Haddad, 1992), cuja digestibilidade decresceu de $744 \mathrm{~g}$ para $513 \mathrm{~g} \mathrm{~kg}^{-1}$ no período de 20 a 70 dias $\left(4,6 \mathrm{~g} \mathrm{~kg}^{-1} \mathrm{dia}^{-1}\right)$.

Digestibilidade abaixo de $650 \mathrm{~g} \mathrm{~kg}^{-1}$, o que segundo Moore \& Mott (1973) seria um fator limitante do consumo, foi observada a partir de
40 dias de idade. No trabalho de Palhano \& Haddad (1992), este valor foi atingido a partir dos 33 dias de crescimento do cultivar Coastcross-1.

Os teores máximos de FDN e FDA foram observados aos 55 e 70 dias de crescimento (790 $\mathrm{g}$ e $390 \mathrm{~g} \mathrm{~kg}^{-1}$ ), respec-tivamente, segundo as equações de regressão (Figura 1). A variação ocorrida na FDN (acréscimos até 55 dias e posteriores decréscimos) pode ser devida à participação da hemicelulose na fração FDN; Arroyo-Aguilú \& Coward-Lord (1974) não observaram correlação entre a idade da planta e a concentração de hemicelulose em forrageiras tropicais, sendo maior a correlação entre FDA e idade que entre FDN e idade, devido à participação do conteúdo de hemicelulose na fração FDN.

Os teores apresentados por Palhano \& Haddad (1992) são superiores aos deste experimento, considerando as mesmas idades de corte. As concentrações máximas de FDN e FDA em Coastcross-1 ocorreram aos 70 dias de idade (806 g e $464 \mathrm{~g} \mathrm{~kg}^{-1}$ ). No trabalho de Gomide (1996), as concentrações máximas de FDN e FDA em Florakirk ocorreram aos 56 e 70 dias de crescimento (820 g e $468 \mathrm{~g} \mathrm{~kg}^{-1}$ ), respectivamente. $\mathrm{O}$ aumento na concentração de FDN e FDA ocorreu tanto na fração folha como na haste, sendo mais acentuado na primeira.

Gomide (1996) observou um decréscimo na relação folha:haste com o aumento da idade de corte de Florakirk (de 0,93 para 0,29, no período de 20 a 70 dias) e uma redução no valor nutritivo de ambas as frações, sendo mais acentuada nas hastes.

O teor de proteína bruta decresceu linearmente $(P<0,05)$ entre 20 e 70 dias de idade (de $176 \mathrm{~g}$ para $69 \mathrm{~g} \mathrm{~kg}^{-1}$ ). A concentração mínima de $\mathrm{PB}$ necessária para que não haja redução no

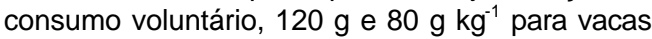
em lactação e bovinos de corte, segundo Forbes (1995), seria atendida até os 40 e 60 dias de crescimento, respectivamente. Postiglioni \& Messias (1998) avaliaram o potencial forrageiro da Florakirk no Estado do Paraná e observaram, em todas as estações de crescimento, teores de PB acima da exigência mínima animal quando a gramínea foi submetida a corte aos 45 dias de crescimento.

As concentrações dos macronutrientes ( N, P, K, Ca, Mg e S) diferiram em função a idade da planta (TABELA 1). Em geral, os teores dos macronutrientes decresceram com o avanço da idade, com exceção dos de $S$, que permaneceram constantes até 60 dias. 
Segundo Gomide (1978), a queda nos teores dos minerais com o avanço da maturidade da planta deve-se, provavelmente, ao efeito de diluição do mesmos na matéria seca produzida e acumulada. Outros fatores também podem contribuir para isto: alterações na relação folha:haste, morte de folhas senescentes, diversidade no padrão de absorção dos minerais ao longo do ciclo da cultura e sua redistribuição entre os vários órgãos da planta, reduzindo assim a concentração dos minerais na planta inteira (Norton, 1982; Fleming, 1973).

As concentrações de $\mathrm{N}, \mathrm{K}$ (entre 30 e 60 dias), Ca e $S$ estão dentro dos intervalos considerados normais por Gallo et al. (1974) para gramíneas tropicais no Estado de São Paulo. Os teores de $\mathrm{P}, \mathrm{Mg}$ e de $\mathrm{K}$ (apenas aos 20 dias) seriam classificados como acima do normal; estando abaixo do normal apenas a concentração de $\mathrm{K}$ aos 70 dias. Esta elevada concentração de alguns macronutientes observada em Florakirk pode ser devida ao efeito de concentração dos minerais na planta em função do crescimento limitado da mesma. Em Coastcross-1 (Palhano \& Haddad, 1992), de modo geral, a concentração de macronutrientes foi inferior à observada em Florakirk, embora as concentrações dos mesmos também tenham decrescido com o avanço da idade da planta.

Conforme as recomendações de National Research Council $(1989,1996)$, os teores de macronutrientes no cultivar Florakirk seriam adequados à nutrição de bovinos de leite até os 60 dias de idade da planta e de bovinos de corte em todas as idades analisadas, com exceção do $\mathrm{Ca}$ (insuficiente em todas as idades) e de vacas em início da lactação (categoria com maiores exigências). $\mathrm{O}$ teor de $\mathrm{P}$ aos 70 dias seria insuficiente apenas para animais com ritmo de crescimento muito acelerado.

O conteúdo dos macronutrientes analisados variou com a idade de crescimento de forma quadrática $(P<0,05)$, assim como a produção de matéria seca. A máxima concentração de $\mathrm{N}$ e de $\mathrm{Ca}$ ocorreu, segundo equações de regressão, aos 54 dias, com 2,72 ge $0,53 \mathrm{~g} \mathrm{~m}^{-2}$, enquanto de $\mathrm{K}, \mathrm{S}, \mathrm{P}$ e $\mathrm{Mg}$ ocorreu aos $48,56,61$ e 70 dias, com 3,44 g; 0,43 g; 0,69 $\mathrm{g} \mathrm{e} 0,53 \mathrm{~g} \mathrm{~m}^{-2}$, respectivamente.

As concentrações dos micronutrientes $\mathrm{B}, \mathrm{Cu}, \mathrm{Fe}, \mathrm{Mn}$ e $\mathrm{Zn}$ estão apresentadas na TABELA 2.

Com exceção do $B$, as concentrações dos demais micronutrientes variaram em função da idade da planta $(P<0,05)$. A variação na concentração de micronutrientes com o aumento da idade foi menor que a observada nos macronutrientes, não apresentando um comportamento típico. Segundo Underwood (1971), não há declínios consideráveis na concentração de micronutrientes com o avanço da maturidade e podem ocorrer mudanças não consistentes com o estádio de crescimento.

As concentrações de micronutrientes em Florakirk estão de acordo com os intervalos considerados normais por Gallo et al. (1974).

Apenas os teores de $\mathrm{Cu}$ e $\mathrm{Zn}$ seriam insuficientes para atender às exigências de bovinos de leite e corte, de acordo com NRC (1989, 1996), o que também foi observado em outras gramíneas no Estado de São Paulo (Gallo et al., 1974).

O valor nutritivo da Florakirk obtido neste trabalho foi superior ao observado por Gomide (1996), no mesmo cultivar, e por Palhano

TABELA 1 -Teores de macronutrientes da matéria seca $\left(\mathrm{g} \mathrm{kg}^{-1}\right)$ da parte aérea de Cynodon dactylon (L.) Pers. cv. Florakirk, em função da idade de crescimento(1).

\begin{tabular}{|c|c|c|c|c|c|c|}
\hline \multirow{2}{*}{$\begin{array}{l}\text { Dias após o } \\
\text { rebaixamento }\end{array}$} & $\mathrm{N}$ & $P$ & K & $\mathrm{Ca}$ & $\mathrm{Mg}$ & S \\
\hline & \multicolumn{6}{|c|}{ - } \\
\hline 20 & $28,2 \mathrm{a}$ & $6,3 \mathrm{a}$ & $31,3 \mathrm{a}$ & $3,8 \mathrm{ab}$ & $3,0 \mathrm{a}$ & $2,4 \mathrm{a}$ \\
\hline 30 & $21,9 \mathrm{~b}$ & $4,8 \mathrm{~b}$ & $25,1 \mathrm{~b}$ & $4,3 \mathrm{a}$ & $3,0 \mathrm{a}$ & $2,5 \mathrm{a}$ \\
\hline 40 & $17,9 \mathrm{c}$ & $4,3 \mathrm{bc}$ & $24,9 b$ & $3,4 \mathrm{~b}$ & $2,9 a$ & $2,3 \mathrm{a}$ \\
\hline 50 & $16,1 \mathrm{c}$ & $4,0 \mathrm{C}$ & $20,7 \mathrm{c}$ & $3,5 \mathrm{~b}$ & $2,5 \mathrm{bc}$ & $2,5 \mathrm{a}$ \\
\hline 60 & $14,1 d$ & $3,9 \mathrm{c}$ & $15,0 d$ & $2,2 \mathrm{c}$ & $2,8 a b$ & $2,3 \mathrm{a}$ \\
\hline 70 & $11,0 \mathrm{e}$ & $3,0 \mathrm{~d}$ & $8,7 \mathrm{e}$ & $2,2 \mathrm{c}$ & $2,4 \mathrm{c}$ & $1,6 \mathrm{~b}$ \\
\hline C.V. (\%) & 4,4 & 12,1 & 11,6 & 9,1 & 7,0 & 18,4 \\
\hline
\end{tabular}

(1) médias seguidas de mesma letra, na mesma coluna, não diferem pelo teste t de Student $(P>0,05)$. 
TABELA 2 - Teores de micronutrientes na matéria seca $\left(\mathrm{mg} \mathrm{kg}^{-1}\right)$ da parte aérea de Cynodon dactylon (L.) Pers. cv. Florakirk, em função da idade de crescimento(1) $^{(1)}$

\begin{tabular}{lccccc}
\hline \multirow{2}{*}{$\begin{array}{c}\text { Dias após o } \\
\text { rebaixamento }\end{array}$} & $\mathrm{B}$ & $\mathrm{Cu}$ & $\mathrm{Fe}$ & $\mathrm{Mn}$ & $\mathrm{Zn}$ \\
\cline { 2 - 6 } & - & & & \\
\hline 20 & $9,7 \mathrm{a}$ & $4,3 \mathrm{bc}$ & $131,3 \mathrm{a}$ & $91,1 \mathrm{ab}$ & $36,5 \mathrm{a}$ \\
30 & $7,7 \mathrm{a}$ & $6,3 \mathrm{a}$ & $118,8 \mathrm{a}$ & $118,1 \mathrm{a}$ & $29,0 \mathrm{~b}$ \\
40 & $7,3 \mathrm{a}$ & $5,9 \mathrm{a}$ & $78,8 \mathrm{~b}$ & $109,7 \mathrm{ab}$ & $26,4 \mathrm{bc}$ \\
50 & $7,2 \mathrm{a}$ & $5,4 \mathrm{ab}$ & $65,6 \mathrm{~b}$ & $81,5 \mathrm{~b}$ & $29,8 \mathrm{~b}$ \\
60 & $6,9 \mathrm{a}$ & $3,7 \mathrm{c}$ & $74,8 \mathrm{~b}$ & $86,6 \mathrm{ab}$ & $30,4 \mathrm{~b}$ \\
70 & $8,7 \mathrm{a}$ & $4,2 \mathrm{bc}$ & $89,5 \mathrm{~b}$ & $84,5 \mathrm{ab}$ & $23,2 \mathrm{c}$ \\
\hline C.V. (\%) & 14,6 & 17,8 & 18,9 & 25,2 & 11,5 \\
\hline
\end{tabular}

(1) médias seguidas de mesma letra, na mesma coluna, não diferem pelo teste t de Student $(P>0,05)$.

\& Haddad (1992), com o cultivar Coastcross-1, quando comparados na mesma idade. Porém, as produções de matéria seca obtidas neste trabalho foram inferiores. Esta superioridade no valor nutritivo deve-se, provavelmente, a um crescimento limitado da planta em função da ocorrência de doença ou de algum fator ambiental limitante ao seu desenvolvimento adequado. Segundo Van Soest (1994), a limitação no crescimento da planta promove o atraso na sua maturação e redução no decréscimo do valor nutritivo esperado com o avanço da idade.

\section{CONCLUSÕES}

Aos 40 dias de crescimento, obser vouse a melhor combinação entre produção de forragem e valor nutritivo, principalmente quanto à concentração de proteína bruta e digestibilidade, sendo essa a idade mais adequada para o corte do cultivar Florakirk, nas condições do presente experimento. Os teores de alguns minerais, no entanto, estariam aquém do mínimo considerado adequado para animais consumindo esta forragem, havendo portanto a necessidade de suplementação. Entretanto, a manutenção de níveis aceitáveis de digestibilidade, FDN e FDA em Florakirk, com estádio de maturação avançado, permitiria a utilização desta forrageira em sistemas de produção onde o diferimento dos pastos seja adotado como técnica de reserva forrageira para o período crítico.

Apesar do elevado valor nutritivo da forragem cortada aos 20 e 30 dias de idade, a produção de matéria seca nestes períodos é limitada.

A suscetibilidade de Florakirk ao ataque de cigarrinhas e a doenças foliares precisa ser avaliada para se determinar a viabilidade da exploração econômica desta forragem.

\section{AGRADECIMENTO}

Ao Sr. Luiz Ermírio de Moraes, proprietário da Fazenda Guará do Pinhal, Itapetininga/SP, pelo apoio dado a este trabalho.

\section{REFERÊNCIAS BIBLIOGRÁFICAS}

ADJEI, M.B.; MISLEVY, P.; KALMBACHER, R.S.; BUSEY, P. Production, quality, and persistence of tropical grasses as influenced by grazing frequency. Proceedings of the Soil and Crop Science Society of Florida, v.48, p.1-6, 1989.

ARROYO-AGUILÚ, J.A.; COWARD-LORD, J. Relationships between and within physical and chemical constituents and in vitro true digestibility in tropical forage grasses. Journal of Agriculture of the University of Puerto Rico, v.58, n.4, p.437-447, 1974.

ASSOCIATION OFFICIAL ANALYTICAL CHEMISTS. Offical methods of analysis. 16.ed. Washington: AOAC, 1995. 1015p. $2 \mathrm{v}$.

BATAGLIA, O.C. Determinação indireta de enxofre em plantas por espectrofotometria de absorção atômica. Ciência e Cultura, v.28, n.6, p.672-675, 1976.

CROWDER, L.V.; CHHEDA, H.R. Tropical grassland husbandry. New York: Longman, 1982. 561p. (Tropical Agriculture Series).

FLEMING, G.A. Mineral composition of herbage. In: BUTLER, G.W.; BAILEY, R.W. Chemistry and biochemistry of herbage. New York: Academic Press, 1973. v.1, p.529-566.

FORBES, J.M. Voluntary food intake and diet selection in farm animals. Wallingford: $C A B$ International, 1995. 532p.

GALLO, J.R.; HIROCE, R.; BATAGLIA, O.C.; FURLAN, P.R.; FURLAN, A.M.C.; MATTOS, H.B.; SARTINI, H.J. FONSECA, M.P. Composição química inorgânica de forrageiras do Estado de São Paulo. Boletim da Indústria Animal, v.31, n.1, p.115-137, 1974. 
GOERING, H.K.; VAN SOEST, P.J. Forage fiber analysis: apparatus, reagents, procedures and some applications. Washington: USDA, 1970. 20p. (Agriculture Handbook, 379).

GOMIDE, C.C.C. Algumas características fisiológicas e químicas de cinco cultivares de Cynodon. Jaboticabal, 1996. 77p. Dissertação (Mestrado) Faculdade de Ciências Agrárias e Veterinárias, Universidade Estadual Paulista "Júlio de Mesquita Filho".

GOMIDE, J.A. Fisiologia do crescimento livre de plantas forrageiras. In: SIMPÓSIO SOBRE MANEJO DE PASTAGENS, 1., Piracicaba, 1973. Anais. Piracicaba: FEALQ, 1973. p.83-89.

GOMIDE, J.A. Mineral composition of grasses and tropical leguminous forages. In: LATIN AMERICAN SYMPOSIUM ON MINERAL NUTRITION RESEARCH WITH GRAZING RUMINANTS, Belo Horizonte, 1976. Proceedings. Gainesville: University of Florida, 1978. p.32-40.

GRIFFIN, J.L.; WATSON, V.H. Production and quality of four bermudagrasses as influenced by rainfall patterns. Agronomy Journal, v.74, n.6, p.1044-1047, 1982.

HOLT, E.C.; CONRAD, B.E. Influence of harvest frequency and season on bermudagrass cultivar yield and forage quality. Agronomy Journal, v.78, n.3, p.433-436, 1986.

MALAVOLTA, E.; VITTI, G.C.; OLIVIERA, S.A. de. Avaliação do estado nutricional das plantas: princípios e aplicações. Piracicaba: Associação Brasileira para Pesquisa da Potassa e do Fosfato, 1989. 201p.

MISLEVY, P.; RUELKE, O.C.; MARTIN, F.G. Grazing evaluation of Cynodon species. Proceedings of Soil and Crop Science Society of Florida, v.47, p.207-212, 1988.

MISLEVY, P.; BROWN, W.F.; KALMBACHER, R.S.; DUNAVIN, L.S.; JUDD, W.S.; KUCHAREK, T.A.; RUELVE, O.C.; NOLING, J.W.; SONODA, R.M. STANLEY JR., R.L. 'Florakirk' bermudagrass. Gainesville: Florida Agricultural Experiment Station, 1995. 9p. (Circular, S 395).

MONSON, W.G.; BURTON, G.W. Harvest frequency and fertilizer effects on yield, quality, and persistence of eight bermudagrasses. Agronomy Journal, v.74, n.2, p.371-374, 1982.

MOORE, J.E.; MOTT, G.O. Structural inhibitors of quality in tropical grasses. In: MATCHES, A.G. Anti-quality components of forages. Madison: CSSA, 1973. p.53-98. (Special Publication, 4).

NATIONAL RESEARCH COUNCIL. Nutrient requirements of beef cattle. 7.ed. Washington: National Academy of Science, 1996. 242p. (Nutrient Requeriments of Domestic Animals, 4).
NATIONAL RESEARCH COUNCIL. Nutrient requirements of dairy cattle. 6.ed. Washington: National Academy of Science, 1989. 157p. (Nutrient Requeriments of Domestic Animals, 3).

NORTON, B.W. Differences between species in forage quality. In: HACKER, J.B. (Ed.) Nutritional limits to animal production from pastures. Farnham Royal: Commonwealth Agricultual Bureaux, 1982. p.89-110.

PALHANO, A.L.; HADDAD, C.M. Exigências nutricionais e valor nutritivo de Cynodon dactylon (L.) Pers. cv. Coast-cross $\mathrm{n} 01$. Pesquisa Agropecuária Brasileira, v.27, n.10, p.1429-1438, 1992.

PEDREIRA, C.G.S. Plant and animal responses on grazed pastures of Florakirk and Tifton 85 bermudagrasses. Florida, 1995. 152p. Thesis (Ph.D.) - University of Florida.

POSTIGLIONI, S.R.; MESSIAS, D.C. Potencial forrageiro de quatro cultivares do gênero Cynodon na região dos Campos Gerais do Paraná. In: REUNIÃO ANUAL DA SOCIEDADE BRASILEIRA DE ZOOTECNIA, 35., Botucatu, 1998. Anais. Botucatu: SBZ, 1998. p.439.

RAYMOND, W.F. The nutritive value of forage crops. Advances in Agronomy, v.21, p.1-108, 1969.

REMY, V.A.; CACERES, O.; GARCIA-TRUJILLO, R.; ESPERANCE, M. Hierba bermuda (Cynodon dactylon L. Pers.). Pastos y Forrajes, v.2, n.1, p.1-46, 1979.

SAS INSTITUTE. User's guide: statistics. release 6.03. Cary, 1988. 1028p.

TILLEY, J.M.A.; TERRY, R.A. A two stage technique for the in vitro digestion of forage crops. Journal of the British Grassland Society, v.18, p.104-111, 1963.

TINNIMIT, P. Forage evaluation using various laboratory techniques. East Lansing, 1974. Thesis (Ph.D.) - Michigan State University.

UNDERWOOD, E.J. Trace elements in human and animal nutrition. 3.ed. New York: Academic Press, 1971. 543p.

VAN SOEST, P.J. Nutritional ecology of the ruminant. 2.ed. New York: Cornell University Press, 1994. 476p.

VICKERY, P.J. Pasture growth under grazing. In: MORLEY, F.H.W. (Ed.) Grazing animals. New York: Elsevier, 1981. p.55-77. (World animal science, 1).

VIEIRA, L.M.; GOMIDE, J.A. Estimativa da digestibilidade e do consumo de matéria seca de gramíneas forrageiras tropicais, pela técnica do rúmen artificial. Experientiae, v.10, n.4, p.71-91, 1970.

Recebido para publicação em 04.12.98

Aceito para publicação em 15.07.99 